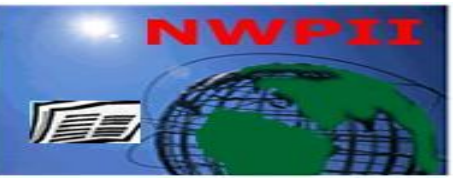

American Journal of Biomedical Sciences

ISSN: 1937-9080

nwpii.com/ajbms

\title{
Window Period for Oxidative Stress Attenuating Intervention (WPOS Theory)
}

\section{Zuolin Zhu}

Sino-US Pficker Pharmaceuticals Co., Ltd, \#85 Nanli Road, Huaibei City, Anhui Province, China

*Corresponding Author:

Zuolin Zhu

Sino-US Pficker Pharmaceuticals Co., Ltd

\#85 Nanli Road

Huaibei City

Anhui Province, China

Email: zuolinzhu@pficker.com.cn or zuolinzhu@yahoo.com

Received: 28 April 2009; $\mid$ Revised: 14 May 2009; | Accepted: 20 May 2009

\begin{abstract}
Clinical trials of antioxidant intervention involving hundreds of human participants have shown almost entirely no positive results or even adverse ones. Other in vitro and animal tests have presented a preponderance of evidence that antioxidant intervention protects cell from damage and is effective in disease prevention. In this review we compared the results from in vitro tests and animal tests with those derived from human clinical trials and found out that there are important differences between them. For in vitro and animal tests, the cells or animals under study either have abnormal levels of oxidative stress before antioxidant intervention or their oxidative stress levels are increased to abnormal levels immediately afterwards. Clinical trials based on data from the general human population gave no benefits or even adverse results, although benefits were observed for clinical trials involving participants with abnormal levels of oxidative stress, such as those with diabetes. I propose a novel theory, "Window Period for Oxidative Stress Attenuating Intervention" (WPOS), that can give a reasonable explanation for the confusing results encountered in the research of oxidative stress up to now. It suggests that antioxidant intervention will play a key role in preventing but not curing disease.
\end{abstract}

Keywords: Antioxidant intervention; oxidative stress; disease prevention; diabetes.

\section{Introduction}

Oxidative stress is a hot research area these days. The yearly rate of new papers published on the topic of oxidative stress has increased exponentially from fewer than 10 papers in 1990 to about 400 papers in 2000 , to more than 5000 papers in 2009 before May 1 [PubMed]. Nine Nobel Prize winners have published their results about oxidative stress [1-9]. Based on the original 
definition of oxidative stress by Sies and the modification by Cutler and Halliwell [10, 11], oxidative stress is the causative factor for many human diseases, as well as for the aging process. Almost all the tests (including animal tests) proved the effectiveness of anti-oxidation treatment in vitro, suggesting that increased consumption of fruits and vegetables rich in antioxidants improve the cardiovascular health of human beings [12-14]. Many clinical trials in humans have produced controversial results [15], especially for the long-term clinical trials involving hundreds of participants $[16,17]$. The results of randomized studies of over 200,000 participants on the effect of vitamin antioxidants published recently [18] revealed that healthy people who take antioxidant supplements increase their risk of early death by as much as 16 percent.

Another randomized, placebo-controlled trial (Selenium and Vitamin E Cancer Prevention Trial [SELECT]) of 35,533 men in the United States, Canada, and Puerto Rico) randomly assigned them to one of four groups (selenium, vitamin $\mathrm{E}$, selenium + vitamin E, and placebo) in a doubleblind fashion. Baseline eligibility included age 50 years or older (African-American men) or 55 years or older (all other men), a serum prostatespecific antigen level of $4 \mathrm{ng} / \mathrm{mL}$ or less, and a digital rectal examination not suspicious for prostate cancer, oral selenium (200 microg/d from L-selenomethionine) and matched vitamin $\mathrm{E}$ placebo, vitamin E (400 IU/d of all rac-alphatocopheryl acetate) and matched selenium placebo, selenium + vitamin $E$, or placebo + placebo for a planned follow-up of minimum of 7 years and a maximum of 12 years. Any prospective participants with prostate cancer and pre-specified secondary outcomes, including lung, colorectal, and overall primary cancer were eliminated from the study. Median overall followup was 5.46 years (range, 4.17-7.33 years). Hazard ratios (99 percent confidence intervals [CIs]) for prostate cancer were 1.13 (99 percent CI, 0.95$1.35 ; \mathrm{n}=473$ ) for vitamin $\mathrm{E}, 1.04$ (99 percent $\mathrm{CI}$, $0.87-1.24 ; \mathrm{n}=432$ ) for selenium, and 1.05 (99 percent CI, $0.88-1.25 ; \mathrm{n}=437$ ) for selenium + vitamin $E$ vs. $1.00(n=416)$ for placebo. There were no significant differences (all $\mathrm{P}>.15$ ) in any other pre-specified cancer end points [19]. Almost all of these kinds of clinical trials were geared toward a normal population and used very safe antioxidants such as vitamins and minerals. These trials gave results of either no effects or adverse effects: reference [20-30] lists some of these trials, except for some clinical trials focusing on specific groups such as persons with diabetes [3134]. In conclusion, almost all these clinical trials using hydrophilic antioxidants gave no-effect results, and hydrophobic antioxidants gave adverse results.

Why have almost all in vitro tests given positive results using antioxidants for disease prevention and extending life span [35, 36] and have even shown benefits in using antioxidant-rich food for human beings, while human clinical trials with pure antioxidants lead to disappointing findings? And why do we see some positive results in the clinical trials of people with diabetes? As some doubts have already appeared about research on oxidative stress, some people think that in spite of substantial evaluation, the preventive effect of antioxidants remains hypothetical and that misleading conclusions are drawn, such as that "some antioxidants have been associated with higher mortality rates"[37]. A reasonable explanation is urgently needed.

Confusion also arises from the fact that the measured oxidative stress levels are almost all higher than zero for healthy individuals. As defined by Dr. Sies, "Oxidative Stress" is "a disturbance in the prooxidant-antioxidant balance in favor of the former, leading to potential damage" [38]. Oxidative stress levels should thus be zero for healthy individuals. Scientists have analyzed derivatized DNA, protein, lipid and carbohydrate as the measurement of damage caused by oxidative stress. They have found that damaged materials are always present even in very healthy cells or animals or human beings in excellent balance.

Comparing analyses of these in vitro and animal tests [10] with clinical trials on human beings, we can see fundamental differences. Oxidative stress was increased to abnormal level for all these in vitro tests and animal tests before antioxidant intervention or oxidative stress was increased to abnormal levels immediately after antioxidant intervention [58-68]. For the general 
population, only about 10 percent of the individuals analyzed appear to have abnormal oxidative stress levels $[35,36]$.

Clinical research reveals that almost all drugs used to control blood glucose level, insulin included, cannot adjust oxidative stress levels to normal [39-42]. Antioxidant interventions for people with diabetes result in positive conclusions because these people have abnormal levels of oxidative stress.

\section{Hypothesis and Discussion}

I believe that antioxidant intervention only has benefits for individuals with abnormal oxidative stress levels. The important application of antioxidant intervention is in the field of disease prevention: to cure disease requires more than just antioxidant intervention. There exists a specific time period for preventing diseases, which I call the "window period for oxidative stress attenuating intervention" (WPOS), a hypothesis that I suggested in 2004 and first published in 2006 [43].

The WPOS theory suggests the following:

1. There is a decisive molecular balance inside the body of living beings. One side of the balance is "reactive species" (abbreviated as RS), and the other side of the balance is "anti-reactive species" (abbreviated as ARS). This balance is a dynamic state. Within the oscillation amplitude of the balance, excess species (either RS or ARS) cause the damage of biomolecules of living organisms, such as DNA, proteins, lipids and carbohydrates. The maximum level of damage is determined by the body's capacity for repair. When the balance is lost, cell damage will be seen in cell dysfunction, cell apoptosis, or cell death.

2. The maximum oscillation amplitude of the balance is one variable of WPOS, and the time span from the moment of diverging from perfect balance status to the moment balance is lost is another.

3. Balance disturbance caused by reactive species in excess results in oxidative stress as traditionally defined. During WPOS, the level of oxidative stress changes from normal (defined as normal oxidative stress level, abbreviated as NOSL), through pseudo-health (defined as pseudo-health oxidative stress level, abbreviated as POSL), to disease-caused (defined as disease-caused oxidative stress level, abbreviated as DOSL) depicted by demonstration diagram Figure 1 below.

The WPOS theory can explain all these controversial results in the research field of oxidative stress noted above, as well as provide guidance on how to design clinical trials for positive results using antioxidant intervention.

For the in vitro tests and animal tests of antioxidant intervention, the hosts (such as cells or animals) are in the oxidative stress level of POSL, their oxidative stress levels are higher than normal induced by chemicals, but lower than DOSL, because cell apoptosis or death will occur at DOSL level [69-71]. Damage caused by excess reactive species is in the range of the host's repair capability. Antioxidants neutralize excess reactive species; there will be no further damage; and the host will repair existing damage in short order. .

The molecular balance is between RS and ARS. When RS is in excess, such as when an oxidant is in excess, excess oxidants will attack biomolecules and cause damage. When ARS is in excess, such as when there is an excess of an antioxidant, the amount of RS is below the requirement. As many living processes require the involvement of reactive species such as energy generation (ATP synthesis), immune reaction and repairing function (activation of nuclear factor $\kappa \mathrm{B})$, and so forth, normal function of life is affected, and damage accumulates, causing cell dysfunction, cell apoptosis and cell death. For example, during physical exercise, energy generation is speeded up and more reactive species generated. Under normal conditions, the body responds to excess reactive species by enhancing its repairing function. Long term physical exercise results in enhanced anti-reactive species capacity. If anti-reactive species were used during physical exercise, reactive species generated would not stimulate a reaction by the body's defense system [57]. 


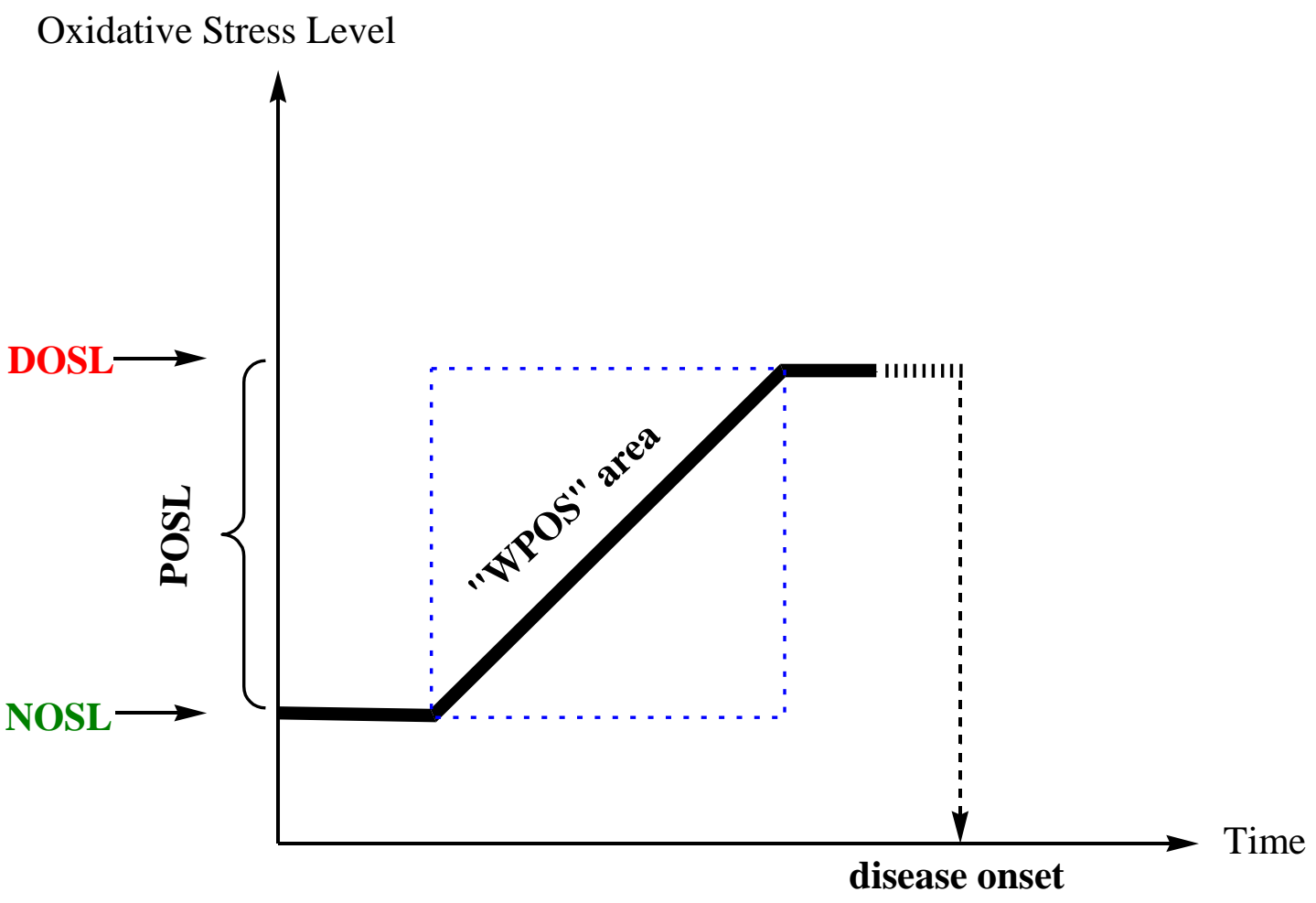

Figure 1. Demonstration diagram of WPOS

1. Rectangle inside dash line is the "WPOS" area

2. X-axial is time, $\mathrm{Y}$-axial is the level of "Oxidative Stress"

3. NOSL $=$ normal oxidative stress level

4. POSL=pseudo-health oxidative stress level

5. DOSL=disease caused oxidative stress level

6. Oxidative stress level stays at DOSL for some time, then disease onset

Since there are only about 10 percent of [11] of individuals who have abnormal oxidative stress levels (in POSL level), clinical trials on general populations will of course give disappointing results. For this 10 percent of individuals, we may obtain positive results from antioxidant intervention. For the remaining 90 percent participants, if hydrophilic antioxidants are used for intervention, clearance of these materials is faster than hydrophobic antioxidants, and only slight adverse responses will be observed. Statistical analysis of these clinical trial data shows no effect. If hydrophobic antioxidants such as vitamin E, beta-carotene, or vitamin A are used for intervention, their clearance inside the body is slow, resulting in an excess of antioxidants. The balance is thus disturbed and statistical analysis of the clinical trial data shows adverse effects. Antioxidant-rich food has much lower levels of antioxidants compared with the dosage used in clinical trials, and digestion takes time that will prevent an antioxidant overdose.

Some researchers have already noticed the existence of WPOS. For example, Cutler and his co-workers found that those individuals who had unusually high oxidative stress status were usually the major responders to antioxidant intervention [11]. Kelly and his colleagues found a lack of 
effect of acute oral ingestion of vitamin $\mathrm{C}$ on arterial stiffness or blood pressure in healthy subjects [44]. Gimeno and his co-workers found that retinol at concentration greater than the physiological limit induces apoptosis in human dermal fibroblasts [45]. Margaritis and Rousseau found that excess antioxidants are harmful, and the benefits of antioxidant supplementation are only exhibited when nutritional status is deficient [46].

The demonstration diagram of WPOS suggests the following conclusions:

1. Disease does not occur at the moment when oxidative stress level reaches DOSL, but when damage caused by excess reactive species reaches the limit of the body's repair capability.

2. At the time of "disease onset," oxidative stress level might drop to some extent, as some damaged cells die at that moment, or some enzyme activity is inhibited.

3. Within the "WPOS" area, an effective method of reducing reactive species can prevent oxidative stress level from reaching DOSL.

Under the WPOS theory, some rules should be applied to antioxidant intervention. One must measure oxidative stress level during the process to arrive at a correct conclusion. First, because certain reactive species cause damage to different cells or different organs, resulting in diseases in varying location, a single vitamin may not be effective in reducing oxidative stress level [46]. If it cannot reach the site where excess reactive species exist, or this single vitamin cannot eliminate the reactive species, the proper material used as anti-reactive species has to be selected. Second, careful selection of clinical trial participants is necessary, as anti-reactive species intervention only works for people with abnormal oxidative stress levels.

Some amazing accomplishments appeared recently having to do with WPOS. Patent PCT/CN2007/071268 revealed a novel way to ease itchiness. With the help of vitamin $\mathrm{E}$, zinc, selenium, and vitamin A, the oxidative stress level of itching skin can be reduced to normal, resulting in itch relief. Itch management without using hormones is important for human health. Another patent, PCT/CN2007/001335, makes known a formulation for lowering the oxidative stress level of respiratory tract to normal. A respiratory tract infected by influenza virus has a higher than normal oxidative stress level. The elevated oxidative stress level makes it possible for the growth of an influenza virus [47]. Once the oxidative stress level of the respiratory tract infected by influenza virus is reduced efficiently to normal, the influenza virus is cleared out and flu is prevented. WPOS thus suggests an effective method for flu prevention.

\section{Conclusion}

WPOS gives a reasonable explanation for the confusions that emerge from the research literature on oxidative stress. Given the limitations inherent in the original definition of "Oxidative Stress," and the confirmation that cell apoptosis or death is governed by the attack of reactive species [48], it seems clear that modifications are needed for the definition of oxidative stress. As the original definition of "oxidative stress" covers only oxidants and antioxidants, many dysfunctions of DNA and proteins caused by the attack of reactive species are not included. Examples include methylation and acetylation of DNA; structure alternation of proteins and DNA caused by the attack of small carbonyl chemicals such as glyoxal, methylglyoxal, malonaldehyde, and monosaccharide, etc; and epigenetic changes caused by ethanol exposure.

Defining the decisive molecular balance of living beings as that maintained between reactive species and anti-reactive species, as opposed to the original balance defined as that between oxidants and antioxidants changes how we think of the causes of disease. It unifies the causes of diseases as depicted by Figure 2. All species that can cause permanent structural alteration of DNA, proteins, lipids and carbohydrates via formation of covalent bond, electron transfer, or other ways are defined as reactive species. Anti-reactive species are those materials that can stop the attack of reactive species toward DNA, proteins, lipids and carbohydrates. The level of oxidative stress has two parts. Part one measures the existed damage of DNA, proteins, lipids and carbohydrates. Part 
two measures the balance between reactive species and anti-reactive species, a potential for damaging biomolecules. Part two needs to measure the total contents of all reactive species, and total concentration of all anti-reactive species. Any concentration of single specie can not represent the level of oxidative stress.

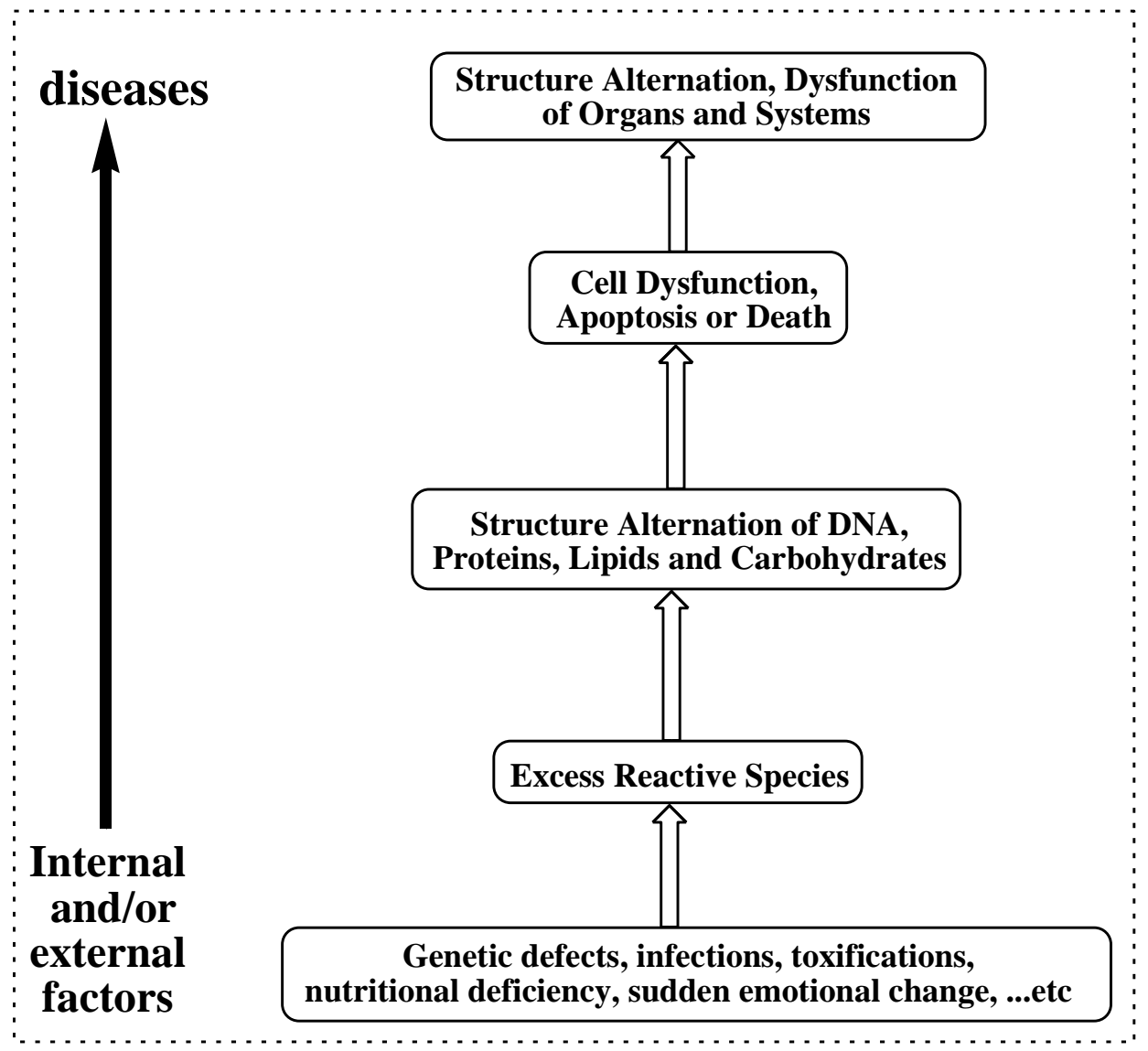

Figure 2. Pathology of Disease under WPOS theory

Based on the research results up to now, excess species are observed inside living beings, whether from internal factors such as genetic defects [49] and immune reactions [50], or from external factors such as infections [51], toxification [52], mechanical damage including (e.g., burning, radiation, freezing) [53], nutritional deficiency [54], or hypoxia. [55].

In conclusion, the promising results from in vitro tests, and disappointing findings from clinical trials, especially those involving more than hundreds of participants, derive from the existence of the WPOS, the window period for oxidative stress attenuating intervention, WPOS.
Patent PCT/CN2005/001555 revealed a formulation for effective control oxidative stress level of diabetes; with normal oxidative stress level, no chronic complications were observed. Antioxidant intervention will be effective for disease prevention within WPOS, but will only show minimum effect in curing diseases, since repairing or clearance is the key issue for curing diseases. Antioxidant intervention can even slow down the healing process of disease, because antibiotics use reactive species to kill bacteria [56] and chemotherapy uses reactive species to kill tumor cells or cancer cells [72, 73]. 


\section{References}

1. Dausset J, et al. In vivo repopulation ability of genetically corrected bone marrow cells from Fanconi anemia patients, PNAS. 2006, 103, 2340-2345.

2. Murad F, et al. Protein Tyrosine Nitration in the Mitochondria from Diabetic Mouse Heart, The Journal of Biological Chemistry, 2003, 278, 33972-33977.

3. Horvitz HR, et al, C. rlegans SIR-2.1 Interacts with 14-3-3 Proteins to Activate DAF-16 and Extend Life Span, Cell, 2006, 125, 11651177.

4. Sharp PA, et al. The Octamer Binding Transcription Factor Oct-1 is a Stress Sensor, Cancer Res., 2005, 65, 10750-10758.

5. Smith M, et al. GDNF Reduces Oxidative Stress in a 6-Hydroxydopamine Model of Parkinson's Disease, Neurosci. Lett., 2007, 412, 259-263.

6. Ciechanover A, et al. Oxidative Stress-related increase in ubiquitination in early coronary atherogenesis, The FASEB Journal, 2003, 17, 1730-1732.

7. Ignarro LJ, et al. The role of oxidative stress in adult critical care, Free Radic. Biol. Med., 2006, 40, 398-406.

8. Kandel ER, et al. Altered Hippocampal Transcript Profile Accompanies an AgeRelated Spatial Memory Deficit in Mice, Learning Memory, 2004, 11, 253-260.

9. Smithies O, et al. Bradykinin B1 and B2 receptors both have protective roles in renal ischemia/reperfusion injury, PNAS, 2007, 104, 7576-7581.

10. Barry Halliwell and John M.C.Gutteridge. Free Radicals in Biology and Medicine, Chapter 9, Oxford: Oxford University Press, 2007.

11. Cutler, RG., Plummer, J, Chowdhury, K, Heward, C. Oxidative Stress Profiling, Ann. N. Y. Acad. Sci., 2005, 1055, 136-158.

12. La Vecchia C, De Carli A, Pagano R. Vegetable consumption and risk of chronic disease, Epidemiology, 1998, 9, 208-210.

13. Rimm EB, Ascherio A, et al. Vegetable, fruit, and cereal fiber intake and risk of coronary heart disease among men, J. Am. Med. Assoc.,
1996, 275, 447-451.

14. Floyd T, Chinchilli VM, et al. Fruit consumption, fitness, and cardiovascular health in female adolescents: the Penn State Young Women's Health Study, Am. J. Clin. Nutr., 1998, 67, 624-630.

15. A)Stephens, N.G. et al. Randomised controlled trial of vitamin $\mathrm{E}$ in patients with coronary disease: Cambridge Heart Antioxidant Study (CHAOS), Lancet, 1996,347, 781-786.

B) Halliwell, B. Establishing the significance and optimal intake of dietary antioxidants: the biomarker concept, Nutr. Rev., 1999, 57, 104 113. C) Lee, C.Y.J. et al. The vascular and antioxidant effects of dark soy sauce in humans, Biochem. Biophys. Res. Commun., 2006, 344, 906-911.

16. Lonn E, Yusuf S, Hoogwerf B, et al. Effects of Vitamin $\mathrm{E}$ on Cardiovascular and Microvascular Outcomes in High-Risk Patients with Diabetes: Results of the HOPE Study and MICRO-HOPE Substudy, Diabetes Care, 2002, 25, 1919-1927.

17. Vivekananthan DP, Sapp S, Penn MS. Use of antioxidant vitamins for the prevention of cardiovascular disease: meta-analysis of randomised trials, Lancet. 2003, 361(9374), 2017-23.

18. Bjelakovic G, Nikolova D, Gluud LL, Simonetti RG, Gluud C. Antioxidant supplements for prevention of mortality in healthy participants and patients with various diseases. Cochrane Database of Systematic Reviews 2008, Issue 2. Art. No.: CD007176.

19. Lippman SM, Klein EA, et al. Effect of selenium and vitamin $\mathrm{E}$ on risk of prostate cancer and other cancers: the Selenium and Vitamin E Cancer Prevention Trial (SELECT), JAMA. 2009, 301(1), 39-51.

20. McKay JD, et al. Vitamin D receptor polymorphisms and breast cancer risk: results from the National Cancer Institute Breast and Prostate Cancer Cohort Consortium, Cancer Epidemiol. Biomarkers Prev. 2009, 18(1), 297-305.

21. Lin $\mathrm{J}$, et al. Vitamins $\mathrm{C}$ and $\mathrm{E}$ and beta carotene supplementation and cancer risk: a randomized controlled trial, J. Natl. Cancer 
Inst. 2009, 101(1), 14-23.

22. Gaziano JM, et al. Vitamins $\mathrm{E}$ and $\mathrm{C}$ in the prevention of prostate and total cancer in men: the Physicians' Health Study II randomized controlled trial, JAMA. 2009, 301(1), 52-62.

23. Zhang SM, et al. Effect of combined folic acid, vitamin B6, and vitamin B12 on cancer risk in women: a randomized trial, JAMA. 2008, 300(17), 2012-21.

24. Aisen PS, Schneider LS, Vicari S, et al. Highdose B vitamin supplementation and cognitive decline in Alzheimer disease: a randomized controlled trial, JAMA. 2008, 300(15), 17741783.

25. Ebbing M, et al. Mortality and cardiovascular events in patients treated with homocysteinelowering B vitamins after coronary angiography: a randomized controlled trial, JAMA. 2008, 300(7), 795-804.

26. Albert CM, et al. Effect of folic acid and B vitamins on risk of cardiovascular events and total mortality among women at high risk for cardiovascular disease: a randomized trial, JAMA. 2008, 299(17), 2027-2036.

27. Jamison RL, et al. Effect of homocysteine lowering on mortality and vascular disease in advanced chronic kidney disease and endstage renal disease: a randomized controlled trial, JAMA. 2007, 298(10), 1163-1170.

28. Cole BF, et al. Folic acid for the prevention of colorectal adenomas: a randomized clinical trial, JAMA. 2007, 297(21), 2351-2359.

29. Bjelakovic G, et al. Mortality in randomized trials of antioxidant supplements for primary and secondary prevention: systematic review and meta-analysis, JAMA. 2007, 297(8), 842857.

30. Bazzano LA, et al. Effect of folic acid supplementation on risk of cardiovascular diseases: a meta-analysis of randomized controlled trials, JAMA. 2006, 296(22), 27202726.

31. Uzi Milman, Shany Blum, et al. Vitamin E Supplementation Reduces Cardiovascular Events in a Subgroup of Middle-Aged Individuals With Both Type 2 Diabetes Mellitus and the Haptoglobin 2-2 Genotype: A Prospective Double-Blinded Clinical Trial, Arterioscler. Thromb. Vasc. Biol.,2008, 28,
341-347.

32. Dan Ziegler, Alexander Ametov, et al. Oral Treatment with $\alpha$-Lipoic Acid Improves Symptomatic Diabetic Polyneuropathy: The SYDNEY 2 trial, Diabetes Care, 2006, 29, 2365-2370.

33. Mark A. Babizhayev, et al. $N$-Acetylcarnosine and histidyl-hydrazide are potent agents for multitargeted ophthalmic therapy of senile cataracts and diabetic ocular complications, Journal of Drug Targeting, 2009, 17, 36-63.

34. Anne Katrin Sjølie, et al. Effect of candesartan on progression and regression of retinopathy in type 2 diabetes (DIRECTProtect 2): a randomised placebo-controlled trial, Lancet, 2008, 372, 1385-1393.

35. Cutler RG. Oxidative Stress Profiling: Part I. Its Potential Importance in the Optimization of Human Health, Ann. N. Y. Acad. Sci., 2005, 1055, 93-135, and references therein.

36. Cai W., et al. Reduced Oxidant Stress and Extended Lifespan in Mice Exposed to a Low Glycotoxin Diet: Association with Increased AGER1 Expression, Am. J. Pathol., 2007, 170, 1893-1902, and references therein.

37. No authors listed. Antioxidants: not for prevention, Prescrire Int. 2009, 18(99), 34, PMID: 19391300.

38. Sies H. Oxidative Stress II. Oxidants and Anti-oxidants. Academic Press: London, 1991.

39. Fumi Sawada, et al. Differential effect of sulfonylureas on production of reactive oxygen species and apoptosis in cultured pancreatic $\beta$-cell line, MIN6, MetabolismClinical and Experimental, 2008, 57, 10381045.

40. Dailey, G. Beyond insulin replacement: addressing the additional needs of the diabetes patient, Diabetes, Obesity and Metabolism, 2008, 10, 83-97.

41. Ge X, et al. Chronic Insulin Treatment Causes Insulin Resistance in 3T3-L1 Adipocytes through Oxidative Stress, Free Radical Res, 2008, 42, 582-591.

42. Raymond Farah, et al. Intensification of oxidative stress and inflammation in type 2 diabetes despite antihyperglycemic treatment, Cardiovascular Diabetology, 
2008, 7, 20.

43. Zhu, Z. Regulating Oxidative Stress in Curing Diabetes. Zhongguo Yiyao Bao (China Pharmaceutical News), June 16 (2006).

44. Kelly RP., et al. Lack of effect of acute oral ingestion of vitamin $\mathrm{C}$ on oxidative stress, arterial stiffness or blood pressure in healthy subjects, Free Radical Research, 2008, 42, 514-522.

45. Gimeno A, et al. Retinol, at concentrations greater than the physiological limit, induces oxidative stress and apoptosis in human dermal fibroblasts, Experimental Dermatology, 2004, 13, 45-54.

46. Margaritis I, et al. Does physical exercise modify antioxidant requirements? Nutrition Research Reviews, 2008, 21, 3-12, and references therein.

47. Albrecht, $\mathrm{T}$, et al. Receptor-Initiated Activation of Cells and Their Oncogenes by Herpes-Family Viruses, J. Invest. Dermatol., 1992, 98, 29S-35S.

48. Seiler A, et al. Membrane Redox State and Apoptosis: Death by Peroxide, Cell Metabolism, 2008, 8, 237-248.

49. A) Kujoth GC, et al. Mitochondrial DNA Mutations, Oxidative Stress, and Apoptosis in Mammalian Aging, Science, 2005, 309, 481484; B) Ralf Morgenstern, Oxidative Stress and Human Genetic Variation, J. Nutr,. 2004, 134, 3173S-3174S.

50. Kim H, et al. Glutathione depletion inhibits dendritic cell maturation and delayed-type hypersensitivity: Implications for systemic disease and immunosenescence, J. Allergy Clin. Immunol., 2007, 119, 1225-1233.

51. Sekhar P. Reddy. The Antioxidant Response Element and Oxidative Stres Modifiers in Airway Diseases, Current Mol. Medicine, 2008, 8, 376-383.

52. Braconi D, et al. Oxidative Damage Mediated by Herbicides on Yeast Cells, J. Agric. Food Chem., 2008, 56, 3836-3845.

53. Güldal Kirkali, et al. Oxidative DNA damage in polymorphonuclear leukocytes of patients with familial Mediterranean fever, Free Radic. Biol. Med., 2008, 44, 386-393.

54. Kalantar-Zadeh K, et al. Nutritional and antiinflammatory interventions in chronic heart failure, Am. J. Cardiol. 2008, 101(11A), 89E-103E.

55. Pavri RS, et al. Further evidence for oxidative damage to hemoglobin and red cell membrane in leukemia, Leuk. Res., 1983, 7, 729-733.

56. Hassett DJ, et al. Bactericidal Antibiotics and Oxidative Stress: A Radical Proposal, ACS Chem. Biol., 2007, 2, 708-710.

57. Ristow M, et al. Antioxidants prevent healthpromoting effects of physical exercise in humans, PNAS, published online before print, May 11, 2009.

58. Khan SA, et al. Protective effect of green tea extract on gentamicin-induced nephrotoxicity and oxidative damage in rat kidney, Pharmacol. Res,2009, 59, 254-262.

59. Shen X, et al. The protective effect of Zizyphus jujube fruit on carbon tetrachloride-induced hepatic injury in mice by anti-oxidative activities, J. Ethnopharmacol. 2009, 122, 555560.

60. Sharma A, et al. Modulatory role of Emblica officinalis fruit extract against arsenic induced oxidative stress in Swiss albino mice, Chem. Biol. Interact. 2009, 180, 20-30.

61. Birch CS, et al. A novel role for vitamin B12: Cobalamins are intracellular antioxidants in vitro, Free Radic. Biol. Med., 2009 May 3.

62. Suchy J, et al. Dietary supplementation with a combination of alpha-lipoic acid, acetyl-Lcarnitine, glycerophosphocoline, docosahexaenoic acid, and phosphatidylserine reduces oxidative damage to murine brain and improves cognitive performance, Nutr. Res. 2009, 29, 70-74.

63. Iida $\mathrm{C}$, et al. Effect of alpha-tocopherol on carbon tetrachloride intoxication in the rat liver, Arch Toxicol. 2009, 83, 477-483.

64. Alleva R, et al. alpha-Lipoic acid supplementation inhibits oxidative damage, accelerating chronic wound healing in patients undergoing hyperbaric oxygen therapy, Biochem. Biophys. Res. Commun. 2005, 333, 404410.

65. Peterhans E. Oxidants and Antioxidants in Viral Diseases: Disease Mechanisms and Metabolic Regulation, J Nutr., 1997, 127, 962S-965S.

66. O'Donovan P, et al, Azathioprine and UVA 
Light Generate Mutagenic Oxidative DNA Damage, Science, 2005, 309, 1871-1874.

67. Hayashi $\mathrm{T}$, et al. 1-Citrulline and 1-arginine supplementation retards the progression of high-cholesterol-diet-induced atherosclerosis in rabbits, Proc. Natl. Acad. Sci. U. S. A. 2005, 102, 13681-13686.

68. Factor VM, et al. Vitamin E reduces chromosomal damage and inhibits hepatic tumor formation in a transgenic mouse model, Proc. Natl. Acad. Sci. U. S. A. 2000, 97, 21969201.

69. Sun KH, et al. Deregulated Cdk5 promotes oxidative stress and mitochondrial dysfunction, J. Neurochem., 2008, 107, 265278.

70. Wu HY, et al. Cannabidiol-induced apoptosis in primary lymphocytes is associated with oxidative stress-dependent activation of caspase-8, Toxcology Applied Pharmacology, 2008, 226, 260-270.

71. Hurh YJ, et al. 2-Hydroxyestradiol Induces Oxidative DNA Damage and Apoptosis in Human Mammary Epithelial Cells, J. Toxic Envir. Health, 2004, 67, 1939-1953.

72. Magwere T, et al. Manipulation of oxidative stress to induce cell death in Ewing's sarcoma family of tumours, European Journal of Cancer, 2008, 44, 2276-2287.

73. Kirshner JR, et al. Elesclomol induces cancer cell apoptosis through oxidative stress, Mol. Cancer Ther., 2008, 7, 2319-2327. 